\title{
Uso de metodologia ativa na disciplina gerenciamento de enfermagem em saúde coletiva da FEO/ UFPEL
}

\section{Using the active methodology in the nursing management discipline of collective health of FEO/ UFPEL}

\section{El uso de la metodologia activa en la disciplina de gerenciamento de enfermaria em salud colectiva de la FEO/ UFPEL}

\author{
Rita Maria Heck', Vanda da Rosa Jardim", Alitéia Santiago Dilélio"I', Solange José da SilvalV \\ 'Enfermeira. Dra. Enfermagem pela Universidade Federal de Santa Catarina. Prof. Adjunto Faculdade de Enfermagem e Obstetrícia da \\ Universidade Federal de Pelotas (FEO/UFPel). Email: feo-pos@ufpel.tche.br. \\ "Enfermeira Dra. Enfermagem/UFSC. Prof. Assistente FEO/UFPel. Email: phein@uol.com.br. \\ II' Enfermeira, Professora Substituta FEO/UFPel. Email: aliteia@gmail.com. \\ Iv Enfermeira. Técnica da Secretaria Estadual de Saúde do Rio Grande do Sul. Email: solange-silva@saude.rs.gov.br.
}

\section{RESUMO}

A preparação de recursos humanos para atuar no Sistema Único de Saúde (SUS) é um desafio para as instituições públicas de ensino superior. O artigo tem o objetivo de descrever a experiência de graduandos de enfermagem no aprendizado do Gerenciamento em Saúde Coletiva aplicando uma metodologia ativa de compreensão do SUS, comentando aspectos positivos e limitadores desse processo. A experiência aconteceu no Curso de Graduação em Enfermagem e Obstetrícia da Universidade Federal de Pelotas/RS, no período de 2000-2008. Como aspecto positivo os acadêmicos destacam a aproximação com o mundo do trabalho na lógica do SUS, utilizando-se de ferramentas como o planejamento, organização, coordenação, compreensão da hierarquização e regionalização dos serviços de saúde no município e região. Neste contato o acadêmico é motivado a exercitar a crítica e reflexão em relação aos indicadores de saúde, à gestão de recursos humanos, físicos e financeiros e tomar decisões indicando o que é prioridade para um contexto específico. Reside aí um limite, os acadêmicos desejam discutir e sugerir uma proposta formulada, visitando o município, o que não foi viabilizado até o momento. O espaço também permite acompanhar mudanças que visam o fortalecimento do SUS a exemplo do Pacto pela Vida.

Descritores: Enfermagem; Gestão em Saúde; Educação; Sistema de Saúde.

\section{ABSTRACT}

The preparation of human resources to serve on the National Health System (SUS) is a challenge to public institutions of higher education. This article aimed to describe the experience of the students in nursing college in the learning of the management in public health applying an active methodology for understanding the SUS, commenting on the merits and limitations of this process. The experience happened in the Nursing and Obstetrics College of the Federal University of Pelotas/RS, from 2000 to 2008 . One positive aspect highlighted by the scholars is the approach to the world of work in the SUS logic, using tools such as planning, organization, coordination, understanding of hierarchy and regionalization of health services in the city and region. In the academic contact is motivated to exercise the criticism and reflection in relation to health indicators, and the management of human resources, physical and financial decisions indicating what a priority for a specific context. There is a limit, the academics want to discuss and suggest a the proposal made by visiting the council, which has not been possible so far. The space also allows monitoring changes which aims to strengthening the SUS following the example of Pact for Life.

Descriptors: Nursing; Management in health; Educación; System of Health.

\section{RESUMEN}

La preparación de los recursos humanos para prestar servicios en el Sistema Único de Salud (SUS) es un desafío a las instituciones públicas de educación superior. El artículo tiene lo objetivo de describir la experiencia de graduandos en enfermería en el aprendizaje del gerenciamiento en salud colectiva aplicando una metodología activa de comprensión del SUS, comentando aspectos positivos y limitadores de eso proceso. La experiencia aconteció en la Facultad de Enfermería e Obstetricia de la Universidad Federal de Pelotas/RS en lo período de 2000-2008. Un aspecto positivo que los académicos destacan es la aproximación con el mundo del trabajo en la lógica del SUS, utilizando herramientas tales como la planificación, organización, coordinación, comprensión de la jerarquía y la regionalización de los servicios de salud en la municipalidad y la región. En este contacto el académico es motivado para ejercer la crítica y reflexión, en relación con los indicadores de salud, la gestión de los recursos humanos, físicos y financieros, indicando lo que es prioridad para un contexto especifico. Hay un límite, los académicos desean debatir y sugerir una propuesta formulada, visitando el municipio, lo que no ha sido posible hasta ahora. El espacio también permite el seguimiento de los cambios que visan fortalecer el SUS, siguiendo el ejemplo del Pacto por la Vida.

Descriptores: Enfermería; Gerencia en salud; Enseñanza; El Sistema de Salud. 


\section{NTRODUÇÃO}

A progressiva implantação/solidificação do Sistema Único de Saúde (SUS) e a necessidade de ensinar sobre o processo de gestão de políticas públicas é um desafio na maioria dos currículos de formação da área da saúde. Nesta perspectiva programas e formas de intervenção para solucionar os problemas de saúde pública passam a ser demandas que necessariamente devem ser discutidos na academia, para auxiliar na organização prática, em busca de serviços mais coerentes, que atendam as decisões do controle social e que discutam as desigualdades sociais ${ }^{(1-2)}$ Cientes deste desafio e entendendo que faz parte do ensino de Administração a abordagem de aspectos inerentes ao planejamento e gestão do SUS, iniciamos no início de 2000, uma experiência junto aos alunos do oitavo semestre da graduação em enfermagem da Faculdade de Enfermagem e Obstetrícia (FEO) da Universidade Federal de Pelotas (UFPel).

A disciplina Gerenciamento de Enfermagem em Saúde Coletiva passou a ser desenvolvida junto a Coordenadoria Regional de Saúde de Pelotas/RS, perfazendo 68 horas práticas; tendo em vista a ementa de "proporcionar o planejamento, execução, acompanhamento e a avaliação dos programas assistenciais em serviços de saúde coletiva". Ao ser idealizada, a disciplina buscou complementar a prática de administração de enfermagem na área de saúde pública, que convencionalmente se restringia à atuação no espaço hospitalar.

A formação do enfermeiro na perspectiva de abranger a saúde coletiva vem sendo discutida desde a década de 80, marcada pelo compromisso com os problemas sociais ${ }^{(3)}$ sugerindo aproximar a formação do profissional com as mudanças em relação aos objetivos da saúde pública brasileira(4). Para que tal movimento se desencadeie o profissional Enfermeiro deve receber subsídios que o levem a compreender o processo de gestão política e técnica, acompanhamento e avaliação das ações em saúde ${ }^{(5-}$ 6).

Portanto, observamos a importância da administração e gerência de enfermagem não apenas na área hospitalar como comentado por diversos autores $^{(7-8)}$. Nas atuais circunstâncias é necessário reforçar a perspectiva da formação do enfermeiro para atuar na gerência da saúde coletiva ${ }^{(2,9)}$, o que vêem ao encontro dos objetivos propostos na disciplina. Embora os estudos citados estejam mais voltados para o enfoque da gerência da assistência de enfermagem, trazem subsídios para indicar caminhos coletivos de gestão, tendo em vista a elaboração de políticas e a operacionalização prática destas, visando à melhoria das condições de saúde da população.

Mas, da onde vem esta convicção? A experiência vivenciada no desenvolvimento da disciplina pode contribuir de forma positiva nas discussões acerca da gestão em saúde? Após oito anos de negociações, proposições e articulações a disciplina Gerenciamento de Enfermagem em Saúde Coletiva reforça como ocupação central o SUS. Neste processo exercitamos uma aprendizagem, que direciona o aluno a aprender sobre SUS pondo sentido no seu fazer. Neste interstício criamos espaços (no Conselho Regional de Saúde e na Coordenadoria Regional de Saúde), adaptamos conteúdos, avaliamos atividades, ouvimos sugestões com o objetivo de solidificar a proposta. Ao longo desta jornada entendemos que o proposto vai ao encontro de uma metodologia que se diferencia da convencional, de apenas repassar conteúdo. O exercício está em problematizar na disciplina conteúdos de gestão relacionados a saúde coletiva $^{(10)}$.

Aprender a aprender ${ }^{(11)}$ o SUS exige e oportuniza ao graduando de Enfermagem questionar indicadores de saúde abordados na epidemiologia; em diferentes realidades, como na unidade de internação, no preenchimento burocrático de um procedimento, na necessidade de realizar visita domiciliar a família, entre outras situações; que tem sentido além do aspecto individual de cuidado, e que ao ser executado nem sempre é pontuado na perspectiva coletiva.

Compreendemos que a metodologia ativa, é um processo, que enfatiza a crítica, discussão e troca de conhecimentos e não apenas a descrição de conceitos. Os graduandos, divididos em pequenos grupos, são orientados a compreender o SUS por meio de leitura e análise de documentos produzidos por determinado município; incentivados a refletirem, dar significado a informação e a questionar os dados descritos. A partir de sua compreensão devem exprimir o seu parecer e propor soluções, considerando os princípios do sistema: universalidade, integralidade, eqüidade e participação social, fundamentais na condução do SUS ${ }^{(12)}$.

O comprometimento do Enfermeiro com a proposição do SUS é uma necessidade eminente, que vem sendo repetidamente discutida ${ }^{(2,4,9)}$. Além disso, são desafios contemporâneos da gestão: superar o modelo tradicional centrado no financeiro e de administração de material, desenvolver linhas diferenciadas com enfoque nas especificidades, superar o reducionismo na atenção a saúde e criar práticas democráticas na dinâmica gerencial ${ }^{(13)}$. Possibilidades que estão ao alcance do enfermeiro que aplica a criatividade e inovação nos serviços de saúde ${ }^{(14)}$

Como enfermeiras temos a dizer que a experiência que relatamos é constantemente desafiadora, dinâmica, está vinculada a um contexto histórico que na nossa compreensão é importante 
retomar.

Para objetivar, recordamos alguns fatos da descentralização da saúde no Rio Grande do Sul, especificamente das Coordenadorias; o ano 2000 aponta como momento de emergência dos Pólos de Capacitação em Saúde liderados pela Escola de Saúde Pública, que tencionou cada Coordenadoria a estruturar o Núcleo de Saúde Coletiva (NURESC). As Universidades foram chamadas a participar destes núcleos, apresentando experiências que pudessem ser compartilhadas em nível macrorregional.

A UFPel foi parceira deste processo e sede de cursos voltados à atenção básica e urgência/emergência. Simultaneamente, as Coordenadorias de Saúde apresentaram um substancial ingresso de Enfermeiros no seu quadro profissional, especialmente na Coordenadoria de Pelotas/RS, passando de três para onze profissionais. Este conjunto de fatores contribuiu para articular estágios voluntários e curriculares, ampliando o campo de atuação profissional com enfoque na gestão da saúde.

Neste contexto a Coordenadoria Regional de Saúde emerge como um espaço propício de aprendizagem, a fim de permitir a vivência da prática, pois se constitui cenário e objeto de construção do SUS; um laboratório "in locu" que permite ao aluno a apreensão de múltiplas dimensões: técnica, política e ideológica.

Entretanto, não podemos relevar as diferenças entre os atores envolvidos - Universidade e Serviços de Saúde - concordamos que os processos de trabalho(15) são distintos, podendo trazer trocas de experiências saudáveis para ambas as instituições. A inserção do ensino na Coordenadoria teve que ser conquistado com zelo e respeito, em alguns momentos não foi totalmente receptivo, por parte de alguns profissionais. Talvez a resistência não fosse apenas em relação a inserção do Universidade e, conseqüente, acolhida aos graduandos, mas sim da dificuldade destes profissionais em acompanhar a política de saúde, que no momento, passava pelo processo de descentralização das ações de saúde do estado e a estruturação incipiente dos municípios, que viviam descompassos devido a peculiaridades locais de gestão e alinhamento das ações em saúde.

A apresentação desses aspectos é fundamental para contextualizar o movimento vivido pelo graduando no referido contexto, pois, esse vem ao encontro do comentário de que na área da saúde a reforma do Estado na década de 1990 coincidiu com a reforma do sistema de saúde que passou a ser único e que teve na descentralização caráter comum, com mudanças do foco tanto do planejamento e execução como também de participação no financiamento, proporcionou o desenvolvimento e proliferação de experiências de novos modelos de gestão dos serviços de saúde ${ }^{(6)}$. Estas experiências eram perceptíveis no espaço da Coordenadoria Regional, pois os graduandos em contato com documentos como o Plano Municipal de Saúde, Relatório de Gestão e Prestação de Contas já aprovadas pelos respectivos Conselhos Municipais de Saúde, discutiam contrastes entre formas de gestão e organização da atenção e como os serviços de saúde eram referenciados.

O facilitador deste processo foi o diálogo direto dos graduandos com os profissionais do estado, muitos deles Enfermeiros, responsáveis por motivar os municípios a estruturar programas de saúde que garantam o alcance de indicadores importantes da Agenda de Saúde, como por exemplo, a Mortalidade Infantil, Mortalidade Materna, Vigilância em Saúde, entre outros. Assim ficava evidente o esforço e a responsabilização de somar conhecimento em relação à orientação sobre a forma de estruturar os Programas no município, e de como poderiam subsidiar estes serviços repassando as informações corretamente com o intuito de dar mais visibilidade às ações de saúde e as competências gerenciais do enfermeiro.

O objetivo deste artigo foi descrever a experiência de graduandos de enfermagem no aprendizado do Gerenciamento em Saúde Coletiva aplicando uma metodologia ativa de compreensão do SUS, comentando aspectos positivos e limitadores desse processo.

\section{METODOLOGIA DE ORIENTAÇÃO DA DI SCI PLI NA}

A disciplina segue uma proposta que passou por várias adaptações, seguindo sugestões dos profissionais que atuam na Coordenadoria, os quais são responsáveis pela descentralização do SUS.

Para aprimorar a linguagem e conhecimento dos acadêmicos iniciam-se as atividades com a organização de seminários intraclasse com as seguintes temáticas: Sistema Único de Saúde, comentando-se os direitos do usuário do SUS, Controle Social e participação na gestão, Planejamento em saúde e Financiamento em saúde. Os seminários inicialmente eram realizados nas dependências da Coordenadoria, abertos a participação de funcionários. Por falta de espaço físico, no momento os alunos estão desenvolvendo as atividades nas dependências da faculdade; estes são incentivados a pesquisar informações nos sites do Ministério da Saúde (MS), do Instituto Brasileiro de Geografia e Estatística (IBGE) e da Secretaria Estadual de Saúde (SES) do Rio Grande do Sul.

Após apresentação e discussões acerca dos temas abordados nos seminários, o aluno é encaminhado a Coordenadoria Regional de Saúde, recebe um histograma organizacional e conhece os 
profissionais que respondem pelos respectivos espaços administrativos e / ou ações de saúde que coordenam. A partir deste momento começa a realizar o Diagnóstico de Saúde, a fim de conhecer a realidade de saúde dos municípios da região sul do Rio Grande do Sul.

$\mathrm{Na}$ Coordenadoria Regional de Saúde, atualmente, composta por 22 municípios, os alunos são subdivididos em trios ou duplas, as quais escolhem um município para construção do diagnóstico, utilizando como fonte de informação o Plano Municipal de Saúde e Relatórios de Gestão (correspondentes ao período de um ano). Além destes documentos é estimulado a buscar informações com os profissionais da Coordenadoria, pesquisar bibliografia na internet, utilizando sites do IBGE, MS, SES.

A vivência intensiva na Coordenadoria faz com que os alunos acompanhem as demandas dos municípios, além de serem convidados a participar em atividades cotidianas como reuniões de discussão de programas, reuniões do Conselho Regional de Saúde, reuniões de pactuação com municípios, reuniões em que estão presentes técnicos do Ministério da Saúde, campanhas de vacinação, entre outros.

Neste estágio o foco de interesse da disciplina é que cada grupo de alunos dialogue sobre as diversidades de saúde dos municípios que estão sendo estudados, salientando contrastes que contribuem para compreender especificidades de seus contextos, seguindo um roteiro para direcionar a coleta de dados baseada em Vaughan e Morrow ${ }^{(16)}$. Desta forma podem observar quanto os dados dos municípios estão atualizados, a qualidade da informação, os programas de saúde que o gestor local adere, o fluxo de recursos, as pactuações realizadas, a melhora dos indicadores e a participação do Conselho Municipal.

A atividade culmina no exercício de identificar 10 agravantes de saúde que tenham observado com base nas informações documentais, isto é, o indicador deve apresentar valor coerente com o problema identificado. Também devem ser salientados os pontos positivos do município observados durante a coleta de dados.

Ao final desta tarefa, os alunos destacam um problema entre os listados e constroem o respectivo plano de ação ${ }^{(17)}$ do enfermeiro para a mudança da situação atual. Neste momento o professor discute com os acadêmicos o que se apresenta como problema prioritário, o mais urgente de ser superado, no sentido de exercitar o planejamento estratégico situacional discutido anteriormente nos seminários.

O plano deve deixar claro como, passo a passo, de acordo com as metas, o Enfermeiro agindo encaminha a situação. O Plano de Ação deve descrever detalhadamente o público alvo, o objetivo da atuação, a meta a ser alcançada, o período para o desenvolvimento da ação e a forma de monitoramento e avaliação do produto final.

O Plano de Ação possibilita ao acadêmico trabalhar aspectos diferentes dos abordados anteriormente, pois o problema, necessariamente, não está relacionado ao seminário, que anteriormente apresentou e tem completo domínio. Assim caso ele não tenha compreendido o que foi exposto pelos colegas ou não conhece como funciona determinado programa terá que se instrumentalizar, ler, se informar junto aos profissionais da Coordenadoria para propor a solução de acordo com a legislação do SUS, como, por exemplo, utilizar a resolução $\mathrm{N}^{\circ} 333^{(18)}$, que versa sobre a criação, reformulação, estruturação e funcionamento dos conselhos de saúde.

Ao final do estágio realizamos um seminário aberto ao Conselho Regional de Saúde e aos funcionários da Coordenadoria, em que cada grupo apresenta os dados coletados e o plano de ação prioritário do município investigado, podendo-se trocar informações e assim ter um panorama do SUS na região.

O aluno ainda pode realizar estágio voluntário, a fim de acompanhar as atividades do Enfermeiro, com o objetivo de compreender o seu papel neste espaço de trabalho.

Vale ressaltar ainda, que a experiência proposta vai ao encontro do que é proposto na Lei de Diretrizes e Bases da Educação(19), de formar enfermeiros que tenham um perfil profissional com competências, habilidades e conhecimentos para atuar no SUS.

\section{REFLETI NDO SOBRE OS ASPECTOS POSI TI VOS E NEGATI VOS DA EXPERI ÊNCI A}

O propósito desta disciplina foi permitir ao aluno, a exemplo de administração hospitalar, discutir conteúdos com ênfase na administração da saúde coletiva.

A questão que ganha destaque no discurso dos graduandos é a dificuldade de objetivamente compreender o SUS, até iniciar o oitavo semestre, pois antes, o SUS apresentava-se apenas como teoria. Ao concluir a experiência da disciplina de Gerenciamento, a opinião dos alunos é de que agora tem noção do que lhes espera ao serem profissionais, ainda sugerem aumentar o tempo de exposição, pois o mesmo apresenta-se curto. O método de discutir, rediscutir esclarecer dúvidas e, especialmente, compreender ao final do estágio que o SUS é um sistema que tem uma lógica diferente, de acordo com a realidade encontrada em cada município, ajuda a compreender a situação local das políticas de saúde.

Os alunos pontuam também que a partir desta 
experiência compreendem a lógica de disposição e distribuição dos leitos hospitalares, dos exames laboratoriais, do custo do cuidado diário. Observam também, a importância do preenchimento adequado das fichas de atendimento ambulatorial, da fatura de cada procedimento, a compreender o financiamento. Esse processo ajuda na discussão dos valores que são envolvidos no custo da saúde, o que os ajuda a perceber que o custo pelo procedimento em nível hospitalar é maior que o valor necessário para investimento na atenção básica, especialmente na promoção da saúde e prevenção de doenças.

Diante destas constatações alguns graduandos referem que a disciplina deveria ser oferecida no início do curso, alegando que permitiria entender o SUS e não apenas decorar o seu conteúdo. No entanto, observamos que neste ponto, as opiniões não são unânimes, pois há os que defendem que conhecer os programas, as ações de saúde e os indicadores no espaço da unidade básica de saúde Ihes permitem compreender a lógica do sistema de saúde, e que isso só é possível por ele ter passado por outras disciplinas anteriormente.

Como principal ponto negativo, os alunos apontam a inviabilidade de se deslocarem até o município, para investigar a realidade, ficando restritos a leitura dos documentos que podem não refletir a realidade local. Entendem que isto fragiliza a sua proposta de diagnóstico e que poderiam estar discutindo o plano de ação com o município, sugerindo ações que fortaleçam o SUS. Esta crítica é pertinente, à medida que, o limite está em operacionalizar o deslocamento ou então encontrar uma alternativa de colocar frente a frente aluno e município.

A experiência deste estágio acadêmico é um processo em construção, pois, estamos sempre repensando sua pertinência, se este traz conhecimentos que contribuam para a formação crítica e reflexiva do enfermeiro. Procuramos estudar conjuntamente com os graduandos portarias e resoluções, mostrando que o SUS passa por transformações, vontades políticas, resistências, superações que estarão sempre presentes no processo de trabalho a que estivermos vinculados.

Este movimento contagia alguns acadêmicos, que após o estágio de Gerenciamento de Enfermagem em Saúde Coletiva se motivam a direcionar trabalhos de conclusão de curso, sob forma de monografias, versando sobre Administração, Gestão e Avaliação em Saúde Coletiva, de forma incipiente se empenham na produção de conhecimentos; que é a contrapartida da Universidade para solidificar o SUS.

Ao nos aproximarmos das propostas expressas nas leis da educação nos motivamos a socializar nossa experiência, que é um pequeno fragmento do currículo do Curso de Enfermagem e Obstetrícia da Universidade Federal de Pelotas/RS, que ainda é compartimentalizado. Apesar disto, acreditamos que é possível transformar a realidade, descobrindo caminhos de ensinar e aprender dentro do que queremos e imaginamos que seja o espaço do Enfermeiro no Sistema de Saúde Brasileiro, dentro de uma perspectiva em que o acadêmico é sujeito e/ou elemento ativo, cidadão com espaço para sugerir e ajudar na condução do espaço de formação.

\section{REFERÊNCI AS}

1. Egry EY, Fonseca RMGS, Oliveira MAC, Bertolozzi MR, Takahashi RF, Ciosak SI et al. Políticas e práticas de saúde rumo à equidade: uma abordagem geral. Rev. esc. enferm. USP. 2007;41(n.esp): 762-4.

2. Arestrup C, Tavares CMM. A formação do enfermeiro e a gestão do sistema de saúde. Rev. Eletr. Enf. [Internet]. 2008 [cited 2009 mar 09]; 10(1):228-234. Available from: http://www.fen.ufg. br/revista/v10/n1/v10n1a21.htm.

3. Ferro RC, Lourenço LHSC, Filho AJA. Panorama das políticas públicas no setor saúde e a enfermagem na década de 1980. Esc. Anna Nery. 2006;10(3):48793.

4. Ximenes Neto FRG, Sampaio JJC. Gerentes de território na estratégia saúde da família: análise e perfil de necessidades de qualificação. Rev Bras Enferm. 2007;60(6): 687-95.

5. Meira MDD, Kurcgant P. Avaliação da formação de enfermeiros segundo a percepção de egressos. Acta paul. enferm. 2008; 21(4): 556-61.

6. Melo CMM, Santos TA. A participação política de enfermeiras na gestão do Sistema Único de Saúde em nível municipal. Texto contexto-enferm. 2007; 16(3): 426-32.

7. Jorge MSB, Freitas CHA, Nóbrega MFB, Queiroz MVO. Gerenciamento em Enfermagem: um olhar crítico sobre o conhecimento produzido em periódicos brasileiros (2000-2004). Rev Bras Enferm. 2007;60(1): 81-6.

8. Formiga J MM, Germano RM. Por dentro da história: o ensino de Administração em Enfermagem. Rev Bras Enferm. 2005; 58(2): 222-6.

9. Spagnol CA, (Re)pensando a gerência em enfermagem a partir de conceitos utilizados no campo da Saúde Coletiva. Ciênc. saúde coletiva. 2005; 10(1): 119-27.

10. Santos Filho, Barbosa S. Métodos de ensinoaprendizagem na prática docente em enfermagem: abordagens problematizadoras em disciplinas de saúde coletiva. REME: Rev. Min. Enferm. 2004; 8(3): 402-8.

11. Ceccim RB, Feuerwerker LCM. Mudanças na graduação das profissões sob o eixo da integralidade. Cad. Saúde Pública. 2004; 20(5): 1400-10.

12. Bernardino E, Oliveira E, Ciampone MHT. 
Preparando enfermeiros para o SUS: o desafio das escolas formadoras. Rev Bras Enferm. 2006; 59(1): 36-40.

13. Abrahão AL, Martins CM, Geisler A. O sujeito na literatura acadêmica sobre gestão em saúde: notas para a questão da autonomia. Ciênc. saúde coletiva. 2008; 13(1)247-57.

14. Feldman LB, Ruthes RM, Cunha ICKO. Criatividade e inovação: competências na gestão de enfermagem. Rev Bras Enferm. 2008; 61(2):239-42.

15. Ribeiro EM, Pires D, Blank VLG. A teorização sobre processo de trabalho em saúde como instrumental para análise do trabalho no Programa Saúde da Família. Cad. Saúde Pública. 2004; 20(2): 438-46.

16. Vaughaan JP, Morrow RH. Epidemiologia para os Municípios - manual para gerenciamento dos distritos sanitários. São Paulo: Hucitec; 1992.

17. Pierantoni CR. As reformas do estado, da saúde e recursos humanos: limites e possibilidades. Ciênc. saúde coletiva. $2001 ; 6(2): 341-60$

18. Ministério da Saúde; Conselho Nacional de Saúde. Resolução № 333/03 - Aprova as diretrizes para criação, reformulação, estruturação e funcionamento dos Conselhos de Saúde. Diário Oficial da União. Brasília (Brasil): Ministério da Saúde; 2003.

19. Ministério da Educação; Conselho Nacional de Educação. Resolução № 3/01 - Diretrizes curriculares nacionais do curso de graduação em Enfermagem. Brasília (Brasil): Ministério da Saúde; 2001.

Artigo recebido em 10.01.08.

Aprovado para publicação em 25.05.09. 